\section{$\underset{\substack{\text { hommes } \\ \text { \& migrations }}}{ }$}

\section{Hommes \& migrations}

Revue française de référence sur les dynamiques

migratoires

1307 | 2014

L'Afrique qualifiée dans la mondialisation

\title{
Great Black Music
}

\section{François Bensignor}

\section{CpenEdition \\ Journals}

\section{Édition électronique}

URL : http://journals.openedition.org/hommesmigrations/2927

DOI : 10.4000/hommesmigrations.2927

ISSN : 2262-3353

\section{Éditeur}

Musée national de l'histoire de l'immigration

\section{Édition imprimée}

Date de publication : 1 juillet 2014

Pagination : 194-200

ISBN : 978-2-919040-28-5

ISSN : $1142-852 X$

\section{Référence électronique}

François Bensignor, «Great Black Music », Hommes \& migrations [En ligne], 1307 | 2014, mis en ligne le 15 janvier 2015, consulté le 22 septembre 2020. URL : http://journals.openedition.org/ hommesmigrations/2927; DOI : https://doi.org/10.4000/hommesmigrations.2927 


\title{
MUSIQUES
}

\section{GREAT BLACK MUSIC}

\author{
FRANÇOIS BENSIGNOR
}

L 'exposition consacrée aux musiques noires conçue par Mondomix et produite par la Cité de la musique a remporté un franc succès à Paris du 11 mars au 24 août 2014. Un succès médiatique, avec une riche couverture dans la presse, à la radio, à la télévision. France Ô, partenaire de l'exposition, a notamment produit et diffusé de nombreux programmes originaux sur le sujet. Et surtout un succès public avec une fréquentation très dense jusque dans les derniers jours, totalisant 80 ooo entrées. Retour sur cet événement exceptionnel.

"Exposer la musique est toujours difficile, parce qu'elle est le véhicule émotionnel par excellence, explique le commissaire de l'exposition Marc Benaïche, fondateur de Mondomix. Nous avons cherché à placer la musique au centre, à en faire le cour d'une expérience sensorielle. Afin que le discours auquel elle est rattachée soit le plus juste possible, nous avons voulu respecter la place et la parole de l'artiste. Et nous avons veillé à la justesse du discours historique et musicologique proposé." Muni d'écouteurs branchés sur une tablette tactile de type smartphone, le visiteur est baigné dans le son et l'image. Tout au long de son parcours, il choisit de déclencher tel ou tel document audiovisuel parmi ceux qu'affiche la tablette en fonction de la salle. Une seule visite ne permet pas de consulter les onze heures de programmes audiovisuels... "L'exposition a été conçue comme un assemblage de fragments, dit Marc Benaïche. Chacun d'entre eux pose certaines questions, y apporte des réponses. À partir de sa propre histoire, le visiteur est amené à se questionner. II va saisir des fragments pour élaborer sa construction personnelle ou densifier la conception qu'il avait de ces musiques au préalable." Le smartphone répond à des usages très contemporains de consommation. Lorsqu'il entend une musique qui lui plaît ou l'interpelle, le visiteur peut l'inscrire dans sa propre sélection. Plus tard, en d'autres lieux, il la retrouve sur le site Internet de l'exposition.

\section{Parcours}

Le parcours traverse six espaces thématisés dotés d'une scénographie audiovisuelle particulière. La première salle, "Les légendes des musiques noires", est consacrée aux artistes les plus emblématiques des musiques populaires : Bob Marley, Myriam Makeba, Miles Davis, Nina Simone, Youssou N’Dour, Billie Holiday, Fela Kuti, etc. Autant de stars dont le destin prodigieux, l'œuvre fertile, l'engagement lumineux sont retracés en vidéo. La deuxième, "Mama Africa", questionne la notion de l'Afrique comme berceau du monde. Elle aborde la créativité traditionnelle et contemporaine des cing grandes régions formant le continent: Afrique du Nord, de l'Ouest, de l'Est, centrale, australe et océan Indien. Dans l'espace intitulé "Rythmes et rites sacrés", le spectateur est immergé dans l'univers des cérémonies rituelles réinventées par les anciens esclaves en mémoire des pratiques de leurs ancêtres africains. Soufflant sur des bougies virtuelles, le visi- 
ration de Nelson Mandela en 1990 en écoutant un morceau de Johnny Clegg, etc. "Dans la chronologie, j'ai souhaité redonner la parole aux musiciens, pour montrer qu'eux-mêmes, artistiquement, s'emparent de cette histoire et la commentent, dit Emmanuel Parent. Par exemple, si l'on évoque les pyramides, on pense à l'afro-futurisme de Sun Ra, qui disait être un pharaon venu de la planète Saturne. Au-delà de l'aspect potache de cette posture, il venait réinterroger, avec son humour et sa créativité, l'histoire occidentale, qui se pense comme étant à l'origine de l'Histoire universelle."

Descendant d'un étage, le visiteur pénètre dans la grande salle consacrée aux Amériques noires. Créolisation, recréation, formidable invention, les musiques nées des conflagrations sociales américaines rayonnent par leur richesse extraordinaire. Des jeux d'écrans sont regroupés selon des thématiques géoculturelles, présentant l'essentiel des grands

Fela $\odot$ D.R

teur réanime la transe du candomblé brésilien, du vaudou haïtien, des negro spirituals américains, du maloya réunionnais. II se trouve instantanément cerné par des images de corps emportés par la danse, envahi par des cris et des chants, des tambours lancinants...

Transition didactique après cette expérience des sens, un long couloir propose "un fil historique". "La chronologie n'est pas tant celle des musiques noires que celle du développement d'une identité panafricaine qui s'est souvent dite en musique", explique son auteur Emmanuel Parent, conseiller scientifique de l'exposition. Faisant référence aux pyramides d'Égypte, elle cite Cheikh Anta Diop et permet d'écouter un morceau de Sun Ra. À la fondation de l'Empire mandingue en 1240 est associée Soudiata, pièce maîtresse du répertoire des griots d'Afrique de l'Ouest, dans la version du Rail Band de Bamako jouant sur des instruments modernes. L'implantation de La Nouvelle-Orléans en 1718 est illustrée par la New Orleans Suite de Duke Ellington. On lit le commentaire sur la libé- courants musicaux. Blues, jazz, rock, soul, funk, rap pour l'Amérique du Nord; rumba, mambo, chachacha, puis salsa à Cuba ; mento, ska, reggae, ragga, dancehall en Jamaïque ; gwoka, biguine, zouk dans les Antilles françaises; calypso, soca, rapso à Trinidad; cumbia et tous ses dérivés depuis la Colombie et jusqu'en Argentine, où règne le tango; de la samba à la bossa nova jusqu'au mouvement des tropicalistes brésiliens, etc. Cette salle regorge de témoignages sur la fantastique diversité d'histoires, de transformations, de créations portées par les musiques noires américaines. L'espace "Global Mix", qui clôt le parcours, est consacré aux expressions les plus contemporaines de la sono mondiale. Le rap américain a ses déclinaisons dans tout le monde noir, notamment en Afrique. D'autres courants y sont apparus, comme le coupé-décalé en Côte d'Ivoire ou le kuduro en Angola, tous deux liés à des situations de conflits politiques armés. Impacté par cette masse d'informations audiovisuelles, le visiteur peut se détendre dans des cabines de danse avant de s'en aller. Coaché par un professeur vir- 
tuel, il se filme en action, prestation qu'il peut retrouver sur le site web de l'exposition.

\section{Musiques noires}

Parler de "musiques noires" nous place inéluctablement dans une pensée "raciale", développée à la faveur de la suprématie d'une Europe esclavagiste, aux visées impériales. Le concept même de musiques noires résulte du commerce triangulaire transatlantique. La rupture anthropologique consécutive à la mise en esclavage de populations razziées dans les royaumes et chefferies d'Afrique subsaharienne induisit la notion d'un "peuple noir". Alors que chaque ethnie africaine possède ses langues, ses rites, ses danses, ses musiques, ses dieux, etc., l'entreprise de déshumanisation opérée par les colons au Nouveau Monde réduit toute cette diversité à une couleur de peau. La main- d'œuvre servile achetée aux trafiquants d'êtres humains n'est considérée que pour sa seule force de travail, à l'exclusion de toute pensée ou faculté. Ainsi ne subsiste aux yeux des "Blancs" qu'une masse indifférenciée, celle des "Noirs", la cohabitation des deux engendrant l'espace de la créolité. Les concepts de "culture noire", de "musique noire" dérivent de ce point de vue.

"Définir la culture par la couleur de peau ne peut aboutir qu'à une impasse théorique, qui plus est dangereuse politiquement, insiste l'anthropologue Emmanuel Parent. Toutefois, il n'en reste pas moins que les populations afro-descendantes, en Afrique, en Europe et en Amérique, partagent depuis cinq siècles une histoire commune : celle de la confrontation brutale avec un pouvoir hégémonique européen qui a succombé à l'éblouissement perturbateur que constitue le fait d'être blanc. (...) Commele rappelle l'écrivain haïtien René Depestre, dans l'Antiquité, les Grecs, qui connaissaient et échangeaient avec des peuples africains comme les Égyptiens, 
les Éthiopiens, les Nubiens, ne les ont jamais désignés par la couleur de leur peau. Les Africains euxmêmes, avant l'arrivée des Blancs, ne se vivaient pas comme Noirs. Aussi, d'un point de vue culturel, importe-t-il de souligner que la notion panafricaine de musique noire ne peut exister avant la rencontre coloniale. La pensée raciale binaire est une conception moderne postérieure à cette rencontre. La culture 'noire' doit alors être pensée non pas comme un héritage de la 'superbe Afrique', comme la nommait déjà Baudelaire en 1857, mais comme une construction, une création, qui a rapidement pris forme, dès les débuts de la présence africaine dans le Nouveau Monde, dans le langage des sons : des vibrations bien réelles cette fois' !" Il est intéressant de noter que l'exposition parisienne résulte de l'évolution progressive d'un concept qui s'est d'abord concrétisé en Afrique. C'est à la suite d'un travail réalisé pour l'inauguration du Musée international de l'esclavage à Liverpool en 2007 que Marc Benaïche ressent la nécessité d'une grande exposition autour des musiques noires. Ce projet, évoqué à Salvador de Bahia dans le cadre de l'Année de la France au Brésil, suscite l'intérêt de Jaques Wagner, le gouverneur de l'État de Bahia, puis celui de la star bahianaise Carlinhos Brown. Celui-ci vient d'acquérir un ancien dock situé au pied de la favela de Libertade, pour les habitants de laquelle il figure comme une sorte d'ange tutélaire. Dans ce lieu destiné à accueillir le Museu du Ritmo, l'artiste brésilien envisage de créer avec Marc Benaïche et une équipe d'architectes brésiliens le futur Centro de Música Negra. Lorsque les organisateurs

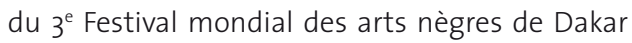
entendent parler du projet, ils demandent à Mondomix d'en réaliser une version temporaire.

"Nous avons eu la chance de réaliser la première exposition multimédia interactive, l'Odyssée des musiques noires, dans la capitale sénégalaise, dit

Marc Benaïche. L'un de nos partenaires brésiliens, l'architecte scénographe Pedro Mendes Da Rocha, a contribué à rénover pour l'occasion la Maison de la culture Douta Seck, institution nationale qui accueillait le projet. Nous avons tenu à apporter aux visiteurs sénégalais un niveau de technicité à la hauteur des plus belles expositions européennes. Le projet s'est ensuite transporté dans le monde créole à La Réunion en 2011, puis en 2012 en Afrique du Sud, pays où la question noire s'est posée de manière exacerbée."

Étrangement, pour sa version parisienne, le titre de l'exposition s'américanise en "Great Black Music", expression forgée en 1965 par Malachi Favors et Lester Bowie, éminents membres de l'Art Ensemble of Chicago et de l'Association for the Advancement of Creative Musicians (AACM). Dans leur esprit, cette notion englobe toutes les musiques dont ils se sentent les héritiers. À cette époque de tension du conflit racial aux États-Unis, il s'agit d'affirmer la grandeur de la musique noire face à ladite "grande musique" occidentale, tout en refusant de recourir à un concept issu de la pensée blanche. Comme l'explique George Lewis, compositeur et tromboniste membre de

L'exposition Great Black Music questionne les origines, la transmission, la transposition, la création, l'évolution des musiques issues des diasporas africaines au Nouveau Monde, jusque dans l'influence que ces nouvelles expressions musicales ont exercée sur la création africaine.

I'AACM : "L'évocation d'une

origine commune, qu'inclut l'idée de 'Great Black Music', s'enracine dans la notion de diaspora panafricaine. Ce lien entre l'histoire de la 'Great Black Music' et l'existence d'une diaspora africaine souligne le fait qu'en dépit de la dispersion des descendants d'esclaves dans de très nombreuses régions, certains traits communs demeurent, qu'ils sont identifiables et qu'ils les unissent." 


\section{MUSIQUES}

\section{Aller-retour}

L'exposition Great Black Music questionne les origines, la transmission, la transposition, la création, l'évolution des musiques issues des diasporas africaines au Nouveau Monde, jusque dans l'influence que ces nouvelles expressions musicales ont exercée sur la création africaine. Schématiquement, après l'abolition de l'esclavage, au cours du XIXe siècle, le développement de nouveaux genres musicaux métis apparaît dans sa pleine force créatrice au Brésil, dans la Caraïbe et aux États-Unis. À la faveur de la modernisation des technologies de reproduction du son et de l'internationalisation de l'industrie de la musique, au début du XXe siècle, la création musicale africaine, sous le régime colonial, est impactée par l'influence de ces musiques transatlantiques cousines d'Amérique. Et à la fin du XXe siècle, l'avènement des musiques du monde est un facteur de multiplication du jeu de miroir entre les deux continents, l'Europe jouant parfois comme troisième bande.

Les exemples foisonnent. Dans le monde créole caribéen se perpétuent notamment des traditions où le tambour, accompagnant des chants à répons, se trouve au centre d'un dispositif festif dans lequel il interagit avec la danse. Certaines formes de chant, de danse ou de jeu de tambour reproduisent à l'évidence des pratiques artistiques africaines relevant de cultures ou de cultes spécifiques. La prééminence des tambours est caractéristique des musiques rituelles intimement liées aux religions syncrétiques apparues au Nouveau Monde après l'abolition de l'esclavage : le vaudou haïtien, la santeria cubaine, le candomblé brésilien. Les tambours y sont généralement joués par groupes de trois, chaque tambour ayant sa propre "personnalité", son timbre, son type de jeu.
En Guadeloupe, le gwoka est une forme emblématique maintenue vivante depuis le XIX siècle. Ses interprètes doivent maitriser la grammaire codée de ses 7 rythmes et des pas de danse qui leur sont associés. Les tambours mènent le jeu : deux "boula" à la sonorité basse et sourde maintiennent le tempo, encadrant le "makè" (marqueur), tambour soliste à la sonorité aiguë, limpide, marquant les variations de la danse et révélant la capacité du soliste à enchaîner les figures au cours de séquences improvisées. On retrouve des formes extrêmement proches du gwoka dans les Petites Antilles : le bèlè en Martinique et à la Dominique, le ka à Sainte-Lucie, le boula et kata à la Grenade et à Carriacou, ou le boula et cutter à Trinidad et Tobago. Le calypso, né dans ce dernier territoire, compte parmi les premières musiques créoles à s'exporter en Amérique du Nord, en Europe, mais aussi en Afrique. Son nom et son style dérivent d'un genre antérieur très populaire, appelé "kaiso". Le phonème "ka" inclus dans ce nom - comme dans gwoka et dans bien d'autres styles cousins de la Caraïbe évoque immédiatement le son de la frappe sur le tambour. Mais, pour certains chercheurs, il est associé à un mot de la langue haussa qui signifie "aller", impliquant la notion de mouvement.

La samba brésilienne est I'héritière directe du "semba" traditionnel angolais. Ce rythme, réexporté, devient un référent de la chanson populaire, adopté comme un style dans le vaste champ de la variété internationale. Puis, popularisé en Europe sous une forme "world" par les interprétations de l'Angolais Bonga, il retrouve une forme beaucoup plus proche de sa nature originelle. Autre exemple, la rumba, danse du "ventre" - selon certains musicologues africains, le mot "cumba", qui désigne le ventre en langue kongo, serait devenu "rumba" à Cuba -, est intimement lié au jeu de tambours rituels conservé dans la mémoire collective des descendants des Kongos déportés à Cuba. Repartie vers l'Afrique, elle 
est copiée-collée par les premiers ensembles de rumba congolaise, alors que se construisent, sur le modèle européen, les nouvelles métropoles de Léopoldville (future Kinshasa) et Brazzaville. Dans les années 1950, les artistes congolais n'hésitent pas à chanter dans une sorte de sabir espagnol assez exotique. Mais les années passant et le génie des leaders de grands orchestres aidant - Joseph "Grand Kallé" Kabasele et l'African Jazz, Franco et l'OK Jazz, Tabu Ley Rochereau et I'Afrisa International-, un style original se forge, à la puissance d'expression telle qu'il va influencer toute la musique moderne de l'Afrique indépendante. C'est sous cette forme que la rumba congolaise débarque par vinyles interposés dans les communautés de Noirs marrons de la côte caraïbe colombienne, où elle donne naissance à la champetta. En Espagne, la rumba cubaine enfante un "palo" dit de "ida y vuelta" (aller-retour) dans le répertoire flamenco, lequel se transforme chez les Gitans de Catalogne en rumba catalane, un genre à part entière. Magnifié par les Gipsy Kings, il se hisse au sommet des Grammy Awards 2014. Peuton dire pour autant que la famille Reyes joue de la "musique noire" ? On n'ira pas jusque-là, même si elle a partagé son trophée avec le chœur sud-africain Ladysmith Black Mambazo : la "world music" permet de ces miracles...

\section{Grammaire universelle}

Il est clair qu'aux États-Unis les premiers rockers jouaient de la musique noire, le rhythm and blues. C'était une stratégie de l'industrie du disque, qui souhaitait engranger des profits dans une confédération d'États américains régie par des lois qui séparaient les races, jusque dans les filières commerciales. Ici se trouve la ligne de démarcation entre les différentes parties du Nouveau Monde. En restant schématique, disons que le monde latin, catholique et syncrétique paraît comme le 


\section{MUSIQUES}

creuset des plus beaux métissages : témoin les splendeurs brésiliennes ou vénézuéliennes et les musiques qui les font danser. Le monde anglo-saxon protestant, libéral mais intolérant, voire sectaire, est pétri d'exclusions, inventeur de la ségrégation raciale, du Klu Klux Klan - aussi de l'apartheid en Afrique du Sud. Quant au monde francophone, il produit le "Tout-Monde" cher à Édouard Glissant, des archipels créoles, qui se vivent isolés, tiraillés entre la tentation du repli et le désir d'avenir.

Qu'ils soient latins, anglo-saxons ou francophones, les imaginaires des empires occidentaux ne considéraient l'Afrique et ses ressortissants qu'à l'image d'un "sous-monde". Le colon blanc n'y voyait qu'un lieu d'exploitation ou de relégation, le purgatoire des fantômes, l'enfer des damnés. Quand il s'intéressait aux richesses culturelles, c'est en pillard qu'il traitait les fétiches et totems, œuvres dédiées à maintenir la proximité entre vivants et ancêtres, afin de perpétuer cet équilibre millénaire entre l'humanité et la nature. Quand l'Amérique lui paraissait comme un Eldorado, territoire des confins de toutes les conquêtes, l'Afrique lui semblait le chaudron des sacrifices, règne de la magie, de la transmutation. Un monde où les dieux vivent entre les humains, en dépit de tout ordonnancement manichéen : c'est dire s'il est dangereux et menaçant pour ceux qui croient en un ordre suprême.

L'avènement de la "world music" dans un monde globalisé libère enfin la parole des artistes. Dès lors qu'elle résonne à travers les réseaux interconnectés, le carcan sémantique de la pensée culturelle raciale idéologisée vole en éclats. Dans la dynamique des flux, la pensée blanche occidentale, avec ses hiérarchies contraignantes, n'a plus de prise sur les échanges. Le processus de décolonisation entraîne l'érosion du mythe du bon sauvage et la musique, en pointe parmi les arts, rend perceptible cet effet. Alors qu'au XX' ${ }^{e}$ siècle les conflits mondiaux ont fait exploser la syntaxe musicale occidentale, la grammaire des musiques noires - le blues, le jazz, la biguine, le calypso, la samba, le chachacha ou le tango - s'est substituée à la vacuité de formules culturelles à bout de souffle.

Rétablissant de fait l'équilibre vital entre la communauté humaine et la connaissance millénaire de la nature, que maintient l'expérience du rite et de sa transmission, la musique noire se trouve immunisée contre toute diabolisation. La fuite occidentale des Européens vers les confins du Nouveau Monde s'est appuyée sur la déportation massive d'Africains, qu'ils tentaient de déshumaniser en les privant de leur culture ancestrale. Effort vain, puisque aujourd'hui des rythmes sophistiqués, élaborés de génération en génération par des communautés, dans des villages de brousse, afin de communiquer avec leurs ancêtres garants des équilibres avec la nature, sont reproduits, recomposés, tout en gardant un sens pour la communauté globale. La "musique noire", avec ses dérivés, devient le commentaire du déplacement des pôles culturels mondiaux. Retournement de situation jamais imaginé par les tenants de la colonisation, l'Afrique aux milliers de langues trouve l'universalité de sa parole à travers son legs à la musique globale. 